\title{
Impaired comprehension of speed verbs in Parkinson's disease
}

Laura J. Speed ${ }^{1}$, Wessel O. van Dam², Priyantha Hirath ${ }^{3}$, Gabriella Vigliocco ${ }^{4}$ \& Rutvik H.

$$
\text { Desai }^{2}
$$

${ }^{1}$ Centre for Language Studies, Radboud University, Nijmegen, Netherlands

${ }^{2}$ Department of Psychology, University of South Carolina, Columbia, USA

${ }^{3}$ School of Medicine, University of South Carolina, Columbia, USA

${ }^{4}$ Experimental Psychology Department, University College London, London, UK

Corresponding author:

Laura J. Speed

Radboud University

Erasmusplein 1

Nijmegen 6500HD

Netherlands

1.speed@let.ru.nl

Word count manuscript text: 5019, Abstract: 196 


\begin{abstract}
Objective: A wealth of studies provide evidence for action simulation during language comprehension. Recent research suggests such action simulations might be sensitive to finegrained information, such as speed. Here we present a crucial test for action simulation of speed in language by assessing speed comprehension in patients with Parkinson's disease (PD). Based on the patients' motor deficits, we hypothesized that the speed of motion described in language would modulate their performance in semantic tasks. Specifically, they would have more difficulty processing language about relatively fast speed than language about slow speed. Method: We conducted a semantic similarity judgment task on fast and slow action verbs in patients with PD and age-matched healthy controls. Participants had to decide which of two verbs most closely matched a target word. Results: Compared to controls, PD patients were slower making judgments about fast action verbs, but not for judgments about slow action verbs, suggesting impairment in processing language about fast action. Moreover, this impairment was specific to verbs describing fast action performed with the hand. Conclusions: Problems moving quickly lead to difficulties comprehending language about moving quickly. This study provides evidence that speed is an important part of action representations.
\end{abstract}

Keywords: embodiment; Parkinson's disease; action semantics; speed 
The view that meaning in language is represented in modality-specific brain regions (e.g., Binder \& Desai, 2011) contrasts with proposals that meaning is stored in abstract, amodal systems (e.g., Landau \& Dumais, 1997). There now exist a large number of studies demonstrating action simulation during language comprehension (for review see Fischer \& Zwaan, 2008). A critical question, however, is the level of abstraction of action simulations; to what extent do they mirror real-world action? Embodied theories are underspecified in terms of how much information is contained in a simulation (Sanford, 2008), or at what grain information is represented.

Action simulations include the specific effector used in the action (Hauk, Johnsrude, \& Pulvermüller, 2004), or the specificity of the action (van Dam, Rueschemeyer, \& Bekkering, 2010). Recent research suggests even fine-grained temporal information is represented in action simulations: whether the action is fast or slow (Speed \& Vigliocco, 2014; Speed \& Vigliocco, 2015; van Dam, Speed, Lai, Vigliocco, \& Desai, in press). Speed \& Vigliocco (2014) showed online simulations are sensitive to speed: when listening to sentences describing fast and slow actions (e.g. The lion dashed to the balloon vs. The lion ambled to the balloon) looking time towards a concurrent visual scene was longer for slow actions compared to fast actions. Related, mental simulation is also sensitive to the degree of effort implied in a sentence (e.g., pushing the piano vs. pushing the chair) (Moody \& Gennari, 2010).

Studies of patients with motor deficits can provide strong tests of causality. If parts of the motor circuit of the brain are crucial to understanding action language, at least some types of deficits in the motor system should lead to difficulties comprehending action language. The present study examines whether action simulations of speed play a crucial role in comprehension of language about speed, by testing impairment in comprehension of fast action in patients with Parkinson's disease (PD) compared to age-matched healthy controls. PD is a neurodegenerative disease caused by a deficiency in the dopaminergic pathway leading to basal 
ganglia atrophy and dopaminergic striatal loss (see Samii, Nutt, Ransom, \& Sampaio, 2004; Rodriguez-Orzo et al., 2009; Helmich, Hallett, Deuschl, Toni, \& Bloem, 2010), resulting in reduced activation in brain areas involved in motor planning and execution, including primary motor cortex and the supplementary motor area (Rascol et al., 1992). PD is characterized by a range of motor problems including bradykinesia and rigidity i.e., slow and difficult movement. We predict that since PD patients move at a slower speed and therefore have difficulty with fast motion, they should similarly be impaired with language about fast motion.

Previous studies have assessed comprehension of action language broadly in patients with motor deficits (e.g. Bak, O'Donovan, Xuereb, Boniface, \& Hodges, 2001; Boulenger, Mechtouff, Thobois, Broussolle, Jeannerod, \& Nazir, 2008; Fernandino et al., 2013a; Fernandino et al., 2013b, but see Kemmerer, Miller, MacPherson, Huber, \& Tranel, 2010; York et al., 2014). Boulenger et al. (2008) found priming effects for action verbs in PD varied as a function of Levodopa uptake (medication improving motor impairment in PD). Fernandino et al. (2013a) removed the grammatical confound in noun-verb comparisons by comparing PD patients and healthy controls on action verb and abstract verb processing. Compared to healthy controls, patients performed worse with action verbs than abstract verbs, reflecting impairment in processing action language rather than the grammatical category of verbs. Kemmerer et al. (2013) suggest that the accuracy results of Fernandino et al. (2013a) can be explained in terms of a slight deficit in action verb comprehension, or as a slight enhancement in abstract verb comprehension. However, their response time results cannot be explained in this manner, as slowing of response time was observed for both action and abstract verbs relative to controls, with greater slowing for action verbs, with a significant interaction. Considering speedaccuracy tradeoff, abstract verb accuracy benefitted from slower response times, but not action verb accuracy even after greater slowing of response time, supporting the conclusion of a specific deficit for action verb comprehension. Fernandino et al. (2013b) also report similar 
results for verbs in sentence contexts, where slowing of response time was observed with a relatively greater effect for action verb sentences, resulting in an interaction. Cardona et al. (2014) found that the action-sentence compatibility effect (ACE) was abolished in early PD patients, but not in patients with peripheral motor deficits. The lack of an ACE effect in PD has been shown to be due to reduced motor potential, aberrant frontotemporal connectivity, and overall volume of basal ganglia atrophy (Melloni et al., 2015). Action language deficits have also been observed in language production: Bocanegra et al. (2015) found disruptions of action verb production in PD patients compared to controls. Moreover, this deficit was unrelated to deficits in executive function or to mild cognitive impairment. Deficiencies in action language have also been observed in spontaneous speech. Using computerized analyses of brief monologues produced by patients and controls, García et al. (2016) found that action-related concepts were less dominant semantic fields in PD discourse than controls (i.e., weighted lower as semantic fields following latent semantic analysis). Thus, several studies have demonstrated that the type of motor system pathology seen in PD can cause specific impairments in action language processing.

Here, we examine whether speed of actions is also represented or simulated during semantic judgments on action verbs. One possibility is that while action concept processing in general is impaired in PD relative to that of abstract concepts, action simulations used in the service of comprehension are not detailed enough to contain information about motion speed, and speed has no effect on comprehension. If speed is part of the simulations, one may expect it to modulate comprehension performance, especially for verbs describing relatively fast movements, because this form of movement is most difficult in PD. Disembodied approaches to language comprehension would predict comprehension of speed is not affected since sensorimotor systems have no functional role in language understanding. 
Here we used a semantic similarity judgment task (SSJ) where participants judged which of two words were most similar to a target word. Words denoted actions similar in speed, i.e. a fast or slow action, or a static action. Actions could be performed with the hand/arm (e.g. grasp) or with the whole body (e.g. run). We compared PD patients with age-matched controls. We predicted an interaction between group (PD vs. control) and verb speed (fast vs. slow).

\section{Method}

\section{Participants}

Eighteen patients with PD were recruited from the Columbia Parkinson's support group and the Palmetto Health Richmond hospital in Columbia, South Carolina. Six patients were removed for having low scores on the Montreal Cognitive Assessment (MoCA) $(\leq 21)$, a cutoff for detecting dementia, leaving 12 patients ( 1 female, $M$ age $=67.4, S D=8.97$ ). All patients were on medication, with an average of 181.56 minutes since last medication (except three patients for whom this information was not recorded). Table 1 presents summary demographics. Fifteen healthy age-matched controls were recruited. Three controls were removed for having a low MoCA score $(\leq 21)$, leaving 12 control participants $(M$ age $=68, S D=9.10, M$ MoCA $=$ 26.92). All participants were paid for their participation. Research was completed according to a protocol approved by the Institutional Review Board of the University of South Carolina.

[Insert Table 1 about here]

\section{Material}

Sixteen fast (e.g. to run), sixteen slow (e.g. to shuffle) and two sets of sixteen verbs of no movement (e.g. to stand) were used in the experiment. Half of the fast and slow verbs were full body actions (e.g. to run) and half were hand/arm movements (e.g. to grasp). Verbs were rated by a separate group of participants in terms of speed ( 1 being very slow, 7 being very fast, 
with an option of "none" available). Fast verbs had an average speed rating of $5.8(S D=0.8$, range $=3.71-6.83$; fast full body $M=6.0, S D=1$, range $=3.71-6.83$; fast hand $M=5.45, S D$ $=0.7$, range $=4.67-5.90)$ and slow verbs had a mean speed rating of $2.7(S D=0.5$, range $1.83-3.43$; slow full body $M=2.83, S D=0.5$, range $=2-3.43$; slow hand $M=2.51, S D=$ 0.5 , range $=1.8-3.20$ ). Verbs were matched in terms of word frequency, number of letters, number of orthographic neighbors, number of phonemes and number of syllables. In a subsequent rating task, a separate group of participants were instructed to rate each verb in terms of the extent to which the arms, hands, legs, feet and torso are involved in the action, and how much effort is required to perform the action (1 being low involvement or low effort, 5 being high involvement or high effort). Full body verbs were rated higher on involvement of legs and feet, and hand verbs were rated higher on involvement of hands and arms, confirming our categorization (see Table 2). Furthermore, verifying our verbs of no movement did sufficiently reflect static action, we found that static verbs were rated as having lower involvement of the arms, hands, and legs than hand verbs, lower involvement of the legs and feet than full body verbs, and as involving less effort than hand verbs and full body verbs (see Table 2). Items were divided into two sets to serve as separate blocks within the experiment. The two sets also reflected the independent variable of speed: fast actions and static actions (fast judgments) and slow actions and static actions (slow judgments). That is, on each trial, a participant had to distinguish fast actions from static actions, or slow actions from static actions. The items were then divided into 32 triplets per block with each item serving as the target, match and foil once.

\section{Procedure}

Participants responded with two colored Ablenet Jelly Bean buttons (www.ablenetinc.com), which is easier than other types of button response (e.g. keyboard press) 
because they are much larger. Participants were instructed to respond with their dominant hand and to rest it between the two buttons between responses.

For each trial, three verbs were presented in a triangular arrangement. Each verb was presented with the word "to" to its left to ensure disambiguation of grammatical class. Participants were to indicate which of the two bottom words was most similar in meaning to the top and to press the right button for the word on the right and the left button for the word on the left. The position of the matching verb was counterbalanced across subjects.

Participants were instructed that verbs would be similar in terms of whether they described movement or not. The stimuli stayed on screen until the participant had responded or the trial had timed out (after 5000ms). Participants were not instructed to respond as quickly as possible, but they were aware that the trial would time out after 5 seconds. Participants first completed six practice trials with words that denoted facial expressions (e.g. to grin) versus words that denoted vocalizations (e.g. to yell) with feedback given on each trial. The task took around 10 minutes to complete.

\section{Results}

Two items were removed because accuracy was $50 \%$ or less in the control group. Individual trials were removed if responses were faster than $250 \mathrm{~ms}$ or outside of $1.5 S D$ of a participant's mean response time (11\% of correct trials: $3 \%$ fast body, $2.7 \%$ slow body, $2.6 \%$ fast hand, 2.7\% slow hand, leaving 1138 trials). One patient was removed for having overall accuracy less than 50\%. Response time analyses were conducted only on accurate trials.

We first conducted $2 \times 2 \times 2$ mixed ANOVAs on accuracy and response time with speed type (fast versus slow) and body part (hand versus full body) as within subjects factors, and group (PD versus control group) as a between subjects factor. Fast verb judgments had higher accuracy than slow verb judgments, $F(1,22)=5.14, p=.03, \eta_{\mathrm{p}}^{2}=.19$, and the control 
group was more accurate than the PD group, $F(1,22)=6.57, p=.02, \eta^{2} \mathrm{p}=.23$. There was also a significant interaction between speed and body part, $F(1,22)=17.94, p<.001, \eta^{2}=.449$, reflecting higher accuracy for slow compared to fast full body verbs, but the opposite pattern for hand verbs. There was no interaction between group and speed or between speed, body part and group, $F<1$, and no effect of body part, $F(1,22)=2.63, p=.12, \eta^{2}=.107$. Mean accuracy is displayed in Figure 1A.

For response time there was no overall difference between the PD group and control group, $F(1,22)=2.24, p=.15, \eta^{2} \mathrm{p}=.092$. Overall, judgments about slow actions took longer than judgments about fast actions, $F(1,22)=6.55, p=.02, \eta^{2}{ }_{p}=.23$, but there was also an interaction between speed and group, $F(1,22)=4.56, p=.04, \eta^{2}=.17$. In line with our prediction, judgments were slower in the PD group than the control group for fast verbs, $t$ (22) $=1.8, p=.04, d=0.77$ (one-tailed), but not slow verbs, $t(22)=1.13, p=.27, d=0.23$, suggesting PD patients have an impairment in comprehending language about fast actions. There was also an interaction between group and body part, $F(1,22)=5.68, p=.02, \eta_{\mathrm{p}}^{2}=.205$, with responses to hand verbs slower than responses to full body verbs in the PD group, $t(11)=$ $2.89, p=.02, d=.198$, but not the control group, $t(11)=.92, p=.38, d=.067$. There was no interaction between speed and body part, $F(1,22)=2.31, p=.14, \eta^{2}{ }_{p}=.095$, or between speed, body part and group, $F(1,22)=1.53, p=.23, \eta^{2}=.065$.

To determine whether age played a role in the two interactions, we reanalyzed the data using an ANCOVA with age as a covariate. With the additional covariate however, the interaction between speed and group was still significant, $F(1,21)=4.52, p=.046, \eta_{\mathrm{p}}^{2}=.177$, as was the interaction between body part and group, $F(1,21)=5.54, p=.028, \eta^{2} \mathrm{p}=.209$. As a second test, we calculated the average difference in response time of patients to fast and slow trials, and the average difference in response time to full body verbs and hand verbs, and then conducted linear regressions on these values with age as a predictor. We found no significant 
effect of age for the speed difference, $\beta=.1, t=.33, p=.75, \mathrm{R}^{2}=.01$, or the body part difference, $\beta=.27, t=.87, p=.75, \mathrm{R}^{2}=.07$. We also conducted regressions with number of years since diagnosis as a predictor. Again the models were not significant, speed difference $\beta=-.53, t=$ $1.26, p=.28, \mathrm{R}^{2}=.29$, body part difference, $\beta=.44, t=1.57, p=.15, \mathrm{R}^{2}=.44$, but the high $\beta$ and $\mathrm{R}^{2}$ values suggest that number of years since diagnosis accounts for a large amount of variance - our data may be underpowered. The pattern suggests that differences in response time between fast and slow trials gets smaller (i.e. responses to fast trials become more impaired) the longer time since PD diagnosis, and by implication, the greater the severity of PD. Similarly, the difference in response time between full body verbs and hand verbs gets larger the longer time since PD diagnosis, suggesting hand verbs are more difficult to comprehend the greater the severity of PD. We further investigated whether cognitive status played any role in effects, but using MoCA score as a predictor of both values was not significant, $\beta=-.2, t=.64, p=.54, \mathrm{R}^{2}=.04 ; \beta=-.09, t=.29, p=.78, \mathrm{R}^{2}=.09$.

Since the first analyses indicated some differences between full body verbs and hand verbs, we further explored the form of speed simulation impaired in PD by looking at verbs describing actions with the whole body and verbs describing actions with the hands separately.

For full-body verbs, there was no difference in accuracy between fast and slow verbs, $F$ $(1,22)=1.25, p=.28, \eta^{2}{ }_{p}=.05$, and no interaction, $F<1$, but there was a marginal effect of group with the PD group having lower accuracy than the control group, $F(1,22)=4.28, p=$ $.05, \eta^{2}{ }_{p}=.16$. Mean accuracy is displayed in Figure 1B. For response time, slow judgments took longer than fast judgments, $F(1,22)=10.45, p=.004, \eta^{2}=.32$, but this time there was no interaction between speed and group, $F<1$, and no difference between PD and the control group, $F(1,22)=1.40, \mathrm{p}=.25, \eta_{\mathrm{p}}^{2}=.06$. Unlike in the first analysis, there was no significant difference between the PD and control group for fast judgments, $t(22)=1.34, p=.2, d=.57$. 
For hand verbs, fast verb trials had higher accuracy than slow verb trials, $F(1,22)=$ $18.56, p<.001, \eta^{2}=.46$, but there was no difference between the control group and the PD group, $F(1,22)=3.84, p=.06, \eta^{2}=.15$, and no interaction between group and speed, $F<1$. Mean accuracy is displayed in Figure 1C. There was no overall difference between fast and slow judgments in response time, $F<1$, and no difference between the PD and control group, $F(1,22)=3.18, p=.09, \eta_{\mathrm{p}}^{2}=.13$, but there was a significant interaction between speed and group, $F(1,22)=5.00, p=.036, \eta^{2} \mathrm{p}=.19$, such that judgments were slower in the PD group than the control group for fast verbs, $t(22)=2.19, p=.04, d=0.93$, but not slow verbs, $t(22)$ $=1.28, p=.21, d=0.54$. Thus, PD patients were specifically impaired at comprehending fast actions performed with the hands, compared to control participants. As before, to determine whether age played a role in this effect, we reanalyzed the data using an ANCOVA with age as a covariate. Again, with the additional covariate the interaction was still significant, $F(1,21)=$ $4.85, p=.04, \eta^{2}{ }_{p}=.19$. We then calculated the average difference in response time of patients to fast and slow trials, and conducted a linear regression on the values with age as a predictor. We found no significant effect of age, $\beta=.11, t=.36, p=.73, \mathrm{R}^{2}=.01$. Again we followed with a regression with number of years since diagnosis as a predictor. The model was not significant, $\beta=-.64, t=1.66, p=.17, \mathrm{R}^{2}=.41$, but again the $\beta$ and $\mathrm{R}^{2}$ values suggest that number of years since diagnosis accounts for a large amount of variance. As before this suggests that responses to fast trials become more impaired the longer time since PD diagnosis. We further investigated whether cognitive status played any role in the effect, but using MoCA score as a predictor of difference in response time between fast and slow judgments was not significant, $\beta=-.06, t=.18, p=.87, \mathrm{R}^{2}=.06$. Figure 2 displays average response time. 


\section{Discussion}

We provide evidence that a fine-grained parameter, speed, is a crucial component of mental simulations of action. Using SSJs on fast and slow action verbs we found PD patients were impaired in judgments about fast actions but not slow actions, reflected in longer response times to make correct judgments. This was specific to verbs describing actions performed with the hand, and not for verbs describing actions performed with the whole body. We believe that the motor symptoms experienced in PD, such as slowness of movement (bradykinesia) and rigidity, place constraints on the mental simulation of fast actions during language comprehension. Reduced motor cortex activation in PD that leads to symptoms such as bradykinesia, also leads to difficulties simulating actions that require speeded movements. The results parallel recent findings (Desai, Herter, Riccardi, Rorden, \& Fridriksson, 2015) where fine-grained parameters of reaching actions were measured in stroke patients. Here, time to perform and initiate the action correlated with speed of processing action verbs and nouns, compared to abstract words.

Why would a deficit in speed processing be found for hand actions but not whole body actions? Although we cannot exclude this difference reflects idiosyncrasies of the patients' motor deficits (i.e. more impairment with hand/arm movement compared to movements with the whole body), a plausible account is that the hand action verbs used here differ to the fullbody verbs in that they depict actions requiring greater precision. Performing a precise action quickly (e.g. grasping) is likely more problematic for a PD patient than performing a whole body action (e.g. walking). Thus, if action simulations mirror real-world action in terms of finegrained features, then PD patients should similarly be more impaired at understanding language about precision actions compared to non-precision actions. Another possibility is that, since participants were using their hands to respond in the task, the interaction between the motor system and mental simulation of action-related meaning becomes more prominent for hand- 
related words (see García \& Ibáñez, 2016, Hand-Action-Network Dynamic Language Embodiment (HANDLE) model for a discussion of situated coupling between the motor and embodied domains). To investigate this possibility, future investigations could implement different response methods, or manipulate the type of ongoing action in the task.

SSJs require explicit semantic processing, and is thus suitable for examining potential semantic deficits. It does not require or encourage artificial mental imagery. If performing mental imagery is part of the process of comprehension and comparison of word meanings, then it is part of conceptual processing. One may ask whether comprehension difficulties for fast speed in PD would be observed for more automatic or shallow language comprehension tasks (e.g. lexical decision with priming c.f. Fernandino et al., 2013a). Recent research suggests simulations are dynamic and context dependent (Lebois et al., 2015), being relied upon more or less in different linguistic and situational contexts. For example, because simulations take time to develop (Barsalou, Santos, Simmons, \& Wilson, 2008), when a quick response is required, lexical associations (statistical information such as word co-occurrence words) are more likely to be recruited than simulations (Louwerse \& Jeuniaux, 2008). It is possible that speed information is a secondary feature of the verbs used here (see van Dam, Speed, Lai, Vigliocco, \& Desai, in press) and simulation may only take into account such features during deeper processing. This is in line with the recent finding that more details are simulated for more explicit semantic tasks (Desai et al., 2015). Fine-grained motor measurements in stroke patients (e.g. action initiation time, movement direction error) correlated with an explicit semantic task (SSJs) but not more implicit tasks (lexical decision, priming), which were correlated with more global action parameters such as total movement time (Desai et al., 2015). Using tasks that manipulate depth of semantic processing could similarly reveal when speed information becomes important. 
We note that elsewhere a specific deficit in action language compared to non-action language was not observed, but a slowing down of comprehension more generally (Kemmerer et al., 2013). Furthermore, both patients and controls responded most slowly and with lowest accuracy to cutting verbs (e.g., cut, slice, hack), which are similar to the present study's "hand verbs" (although this was not found for hitting verbs, which also imply hand actions e.g., hit, poke, jab). However, the PD patients in Kemmerer et al. (2013) were older than the present group (75.5 vs. 67.4 years) and were diagnosed much earlier (7.6 vs. 4.5 years ago). It is therefore likely that the Kemmerer et al. (2013) patients have more cognitive decline, making deficits in action semantics harder to discern. A decline in executive function is especially associated with PD (McKinlay, Grace, Dalrymple-Alford, \& Roger, 2010; Higginson et al., 2003; Levy et al., 2002; Weintraub et al., 2005; Xu et al., 2014). Kemmerer et al. (2013) had a smaller pool of patients $(n=10)$ and half of these patients exhibited mild impairment in executive functions (see their Table 2), potentially diluting any specific effects. Beyond a general decline, according to some theories, comprehension of abstract words rely on verbal associations, and consequently on executive and control mechanisms more, as they lack a direct referent (Schwanenflugel, 1991). This is supported by the activation of the inferior frontal gyrus, an area traditionally associated with executive function and control, for abstract relative to concrete words (Wang, Conder, Blitzer, \& Shinkareva, 2010). Hence, an additional potential factor is that mild executive impairment may specifically affect abstract words, reducing any difference between concrete and abstract words. But on the other hand, our present data suggests effects would be more likely the greater time since diagnosis, at least in the initial phase of the disease. It is possible however, that there is a peak to this effect, after which the executive decline increases sufficiently to make action comprehension difficulties less discernible. MoCA scores did not predict response difference between fast and slow verbs in the present study. However, all of our patients were without mild cognitive impairment and so present an unsuitable 
population in which to test the role of cognitive impairment, instead comparing groups with and without mild cognitive impairment would be more appropriate (e.g., Bocanegra et al., 2017).One interesting finding from Kemmerer et al. (2013) however that supports the present data is that patient's accuracy for cutting verbs correlated with time since diagnosis-with greater disease duration leading to lower accuracy. This is in line with the idea posed above that hand verbs may be more easily affected by motor disorders because they describe actions that require more precision.

York et al. (2014) also found that PD patients did not perform worse on judgments of action verbs compared to cognition verbs, but did find the expected pattern in patients with amyotrophic lateral sclerosis - a condition with atrophy in motor association and prefrontal regions. Of the $22 \mathrm{PD}$ patients in their study however, 14 of them were not cognitively healthy, but ranged from having mild cognitive impairment to dementia. It is unclear to what extent such impairment could differentially affect the different verb types used in their study.

A point to consider in research regarding action-verb processing in motor disorders (see Bak, 2013) is whether deficits should be observed in accuracy measures or response time. In the present study, we observed differences in response time, which is line with studies showing differences in performance on tasks such as lexical decision (Fernandino et al., 2013a; Boulenger et al., 2008) and sentence comprehension (Fernandino et al., 2013b). However, elsewhere differences have been observed in accuracy with semantic similarity judgments (Fernandino et al., 2013a) and verb to picture matching (Bak et al., 2001), for example. One suggestion for this difference is that effects may be observed in accuracy for tasks that are particularly difficult, or when participants are under time pressure (e.g., Rueschemeyer, Lindemann, van Rooij, van Dam, \& Bekkering, 2010). That we find differences in response time but other studies also using semantic similarity judgments find differences in accuracy (Fernandino et al., 2013a) could be due to the difficulty level of the current task being too low, 
or due the lack of an emphasis on responding quickly. Further research manipulating such variables could test these predictions.

There are possible limitations to the present study. First, with only 12 patients, it is possible that our study is underpowered, which could lead to bias in the data (see Button et al., 2013). Investigations involving such populations can often be difficult in terms of participant recruitment, but such issues should be considered. We also note that although our items were matched on various psycholinguistic variables, such as word frequency, there are other variables that may be relevant, such as imageability and semantic-relatedness. For example, it could be possible that pairs of fast verbs were less semantically related than pairs of slow verbs, making the decision more difficult and hence slower. It could be expected though that such a difference between fast and slow verbs in this direction would also be present in the controls, but it was in fact the opposite. Since our pattern of results in PD patients (slower responses to fast verbs) is in the opposite direction to that of controls who were given identical stimuli (slower responses for slow judgments), we are confident that differences do not reflect an unbalanced item set in this sense. Furthermore, intuitively fast judgements should be easier than slow judgments because the task required discriminating motion verbs from static verbs, and fast actions are more different in terms of speed to static actions than slow actions are. It is also possible that the current speed verbs may differ on additional dimensions not considered here. We note for example, that in a follow-up rating task, fast actions were rated as involving more effort than slow actions (3.36 vs. 2.55). It is therefore possible that the present results instead reflect simulation of effort (see Moody \& Gennari, 2010). However, it is unclear to what extent the meaning of effort and the meaning of speed can be disentangled for the present verbs.

It has been suggested that perceptual simulations are schematic (Barsalou, 1999) and thus it is conceivable that they only include salient or coarse details. That evidence exists for simulation of action speed shows that simulations can go beyond a schematic reconstruction of 
action events in general, to a further level of detail, including fine-grained information about the manner of action. By showing that patients with motor problems also have difficulties comprehending language about fast action compared to healthy control participants, we provide evidence that action simulations of speed are a causally involved component in the comprehension of language about speed.

\section{Acknowledgments}

This research was supported by NIH/NIDCD grant R01DC010783 (RHD) and a UCL Bogue Fellowship to L.J. Speed. The paper was written while L. J. Speed was supported by The Netherlands Organization for Scientific Research: NWO VICI grant. We thank Carl Brzorad and Nicholas Riccardi for assistance with running subjects, and Ilja Croijmans for comments on a draft of the paper.

\section{References}

Bak, T. H., O'Donovan D. G., Xuereb, J. H., Boniface, S. \& Hodges, J. R. (2001). Selective impairment of verb processing associated with pathological changes in the Brodman areas 44 and 45 in the motor neurone disease-dementia -aphasia syndrome. Brain, 124, 103-120. doi: 10.1093/brain/124.1.103

Bak, T. H. (2013). The neuroscience of action semantics in neurodegenerative brain diseases: Current Opinion in Neurology, 26(6), 671-677. doi: 10.1097/WCO.0000000000000039

Barsalou, L. W. (1999). Perceptual symbol systems. Behavioral and Brain Sciences, 22(4), $577-609$ 
Barsalou, L.W., Santos, A., Simmons, W.K., \& Wilson, C.D. (2008). Language and simulation in conceptual processing. In M. De Vega, A.M. Glenberg, \& A.C. Graesser, A. (Eds.). Symbols, embodiment, and meaning (pp. 245-283).Oxford: Oxford University Press

Binder, J. R., Desai, R. H., Graves, W. W., \& Conant, L. L. (2009). Where Is the Semantic System? A Critical Review and Meta-Analysis of 120 Functional Neuroimaging Studies. Cerebral Cortex, 19(12), 2767-2796. doi: 10.1093/cercor/bhp055

Bocanegra, Y., García, A. M., Pineda, D., Buriticá, O., Villegas, A., Lopera, F., ... Ibáñez, A. (2015). Syntax, action verbs, action semantics, and object semantics in Parkinson's disease: Dissociability, progression, and executive influences. Cortex, 69, 237-254. doi: 10.1016/j.cortex.2015.05.022

Boulenger, V., Mechtouff, L., Thobois, S., Broussolle, E., C, Jeannerod, M., \& Nazier, T. (2008). Word processing in Parkinson's Disease is impaired for action verbs but not for concrete nouns. Neuropsychologia, 46(2), 743-56. doi:

10.1016/j.neuropsychologia.2007.10.007

Button, K. S., Ioannidis, J. P. A., Mokrysz, C., Nosek, B. A., Flint, J., Robinson, E. S. J., \& Munafo, M. R. (2013). Power failure: why small sample size undermines the reliability of neuroscience. Nature Reviews Neuroscience, 14(5), 365-376.

Cardona, J. F., Kargieman, L., Sinay, V., Gershanik, O., Gelormini, C., Amoruso, L., ...Ibáñez, A. (2014). How embodied is action language? Neurological evidence from motor diseases. Cognition, 131(2), 311-322. doi: 10.1016/j.cognition.2014.02.001

Desai R. H., Herter T., Riccardi N., Rorden C., \& Fridriksson J. (2015). Concepts within reach: Action performance predicts action language processing in stroke. Neuropsychologia, 71, 217-224. Doi: 10.1016/j.neuropsychologia.2015.04.006 
Fernandino, L., Conant, L. L., Binder, J. R., Blindauer, K., Hiner, B., Spangler, K., \& Desai, R. H. (2013a). Parkinson's disease disrupts both automatic and controlled processing of action verbs. Brain and Language, 27(1), 65-74. doi: 10.1016/j.bandl.2012.07.008

Fernandino, L., Conant, L., Binder, J.R., Blindauer, K., Hiner, B., Spangler, K., Desai, R.H. (2013b). Where is the action? Action sentence processing in Parkinson's disease. Neuropsychologia, 51(8), 1510-1517. doi: 10.1016/j.neuropsychologia.2013.04.008

Fischer, M. H., \& Zwaan, R. A. (2008). Embodied language: a review of the role of the motor system in language comprehension. Quarterly Journal of Psychology, 61(6), 825-850. doi: $10.1080 / 17470210701623605$

García, A. M., Carrillo, F., Orozco-Arroyave, J. R., Trujillo, N., Vargas Bonilla, J. F., Fittipaldi, S... M.Cecchi, G. A. (2016). How language flows when movements don't: An automated analysis of spontaneous discourse in Parkinson's disease. Brain and Language, 162, 19-28. doi: 10.1016/j.bandl.2016.07.008

García, A. M., \& Ibáñez, A. (2016). A touch with words: Dynamic synergies between manual actions and language. Neuroscience \& Biobehavioral Reviews, 68, 59-95.

Hauk, O., Johnsrude, I., \& Pulvermüller, F. (2004). Somatotopic representation of action words in human motor and premotor cortex. Neuron, 41(2), 301-7. doi: 10.1016/S0896-6273(03)00838-9

Helmich, R. C., Hallett, M., Deuschl, G., Toni, I., \& Bloem, B. R. (2012). Cerebral causes and consequences of parkinsonian resting tremor: a tale of two circuits? Brain, 135(11), 3206-3226. doi: 10.1093/brain/aws023

Higginson, C. I., King, D. S., Levine, D., Wheelock, V. L., Khamphay, N. O., \& Sigvardt, K. A. (2003). The relationship between executive function and verbal memory in Parkinson's disease. Brain and Cognition, 52(3), 343-352. doi: 10.1016/S02782626(03)00180-5 
Kemmerer, D., Miller, L., MacPherson, M. K., Huber, J., \& Tranel, D. (2013). An investigation of semantic similarity judgments about action and non-action verbs in Parkinson's disease: implications for the Embodied Cognition Framework. Frontiers in Human Neuroscience, 7. doi: 10.3389/fnhum.2013.00146

Landauer, T. K. \& Dumais, S. T. (1997) A solution to Plato's problem: The Latent Semantic Analysis theory of the acquisition, induction, and representation of knowledge. Psychological Review, 104(2), 211-140. doi: 10.1037/0033-295X.104.2.211

Lebois, L. A. M., Wilson-Mendenthal, C. D., \& Barsalou, L. W. (2015). Are automatic conceptual cores the gold standard of semantic processing? The context-dependence of spatial meaning in grounded congruency effect. Cognitive Science, 39(8), 1764-1801. doi: $10.1111 / \operatorname{cogs} .12174$

Levy, G., Jacobs, D. M., Tang, M.-X., Côté, L. J., Louis, E. D., Alfaro, B., ... Marder, K. (2002). Memory and executive function impairment predict dementia in Parkinson's disease: Dementia in Parkinson's Disease. Movement Disorders, 17(6), 1221-1226. doi: $10.1002 / \mathrm{mds} .10280$

Louwerse, M. M. \& Jeuniaux, P. (2008). How fundamental is embodiment to language comprehension? Constraints on embodied cognition. In V.Sloutsky, B. Love, \& K. McRae (Eds.), Proceedings of the 30th Annual Conference of the Cognitive Science Society (pp.1313-1318), Austin, TX: Cognitive Science Society.

Mckinlay, A., Grace, R. C., Dalrymple-Alford, J. C., \& Roger, D. (2010). Characteristics of executive function impairment in Parkinson's disease patients without dementia. Journal of the International Neuropsychological Society, 16(02), 268. doi: $10.1017 / \mathrm{S} 1355617709991299$ 
Melloni, M., Sedeño, L., Hesse, E., García-Cordero, I., Mikulan, E., Plastino, A., ...Ibáñez, A. (2015). Cortical dynamics and subcortical signatures of motor-language coupling in Parkinson's disease. Scientific Reports, 5, 11899. doi: 10.1038/srep11899

Moody, C. L. \& Gennari, S. P. (2010). Effects of implied physical effort in sensory-motor and prefrontal cortex during language comprehension. NeuroImage, 49, 782-793

Rascol, O., Umberto, S., Chollet, F., Celsis, P., Montastruc, J., Marc-Vergnes, J., \& Rascol, A. (1992). Regional cerebral blood flow changes during finger movements and effects of apormorphine. Archives of Neurology 49(2), 144-148

Rodriguez-Oroz, M. C., Jahanshahi, M., Krack, P., Litvan, I., Macias, R., Bezard, E., \& Obeso, J. A. (2009). Initial clinical manifestations of Parkinson's disease: features and pathophysiological mechanisms. The Lancet Neurology, 8(12), 1128-1139.

Rueschemeyer, S.-A., Lindemann, O., van Rooij, D., van Dam, W. \& Bekkering, H. (2010). Effects of intentional motor actions on embodied language processing. Experimental Psychology, 57(4), 260266

Sanford, A. J. (2008). Defining embodiment in understanding. In M. De Vega, A. M.

Glenberg, \& A. Graesser (Eds.), Symbols and embodiment: Debates in meaning and cognition. Oxford, England: Oxford University Press.

Samii, A., Nutt, J. G., Ransom, B. R., \& Sampaio, C. (2004). Parkinson's disease. Lancet, $363,1783-1793$.

Schwanenflugel, P. (1991). Why are abstract concepts hard to understand? In. P.J. Schwanenflugel (Ed.), The psychology of word meaning (pp. 223-250), Mahwah, NJ: Erlbaum

Speed, L. J. \& Vigliocco, G. (2014). Eye movements reveal the dynamic simulation of speed in language. Cognitive Science 38(2), 367-382. doi: 10.1111/cogs.12096 
Speed, L.J. \& Vigliocco, G.(2015). Space, time and speed in language and perception. In Y. Coello, \& M. Fischer (Eds.), Foundations of Embodied Cognition. New York: Psychology Press.

van Dam, W. O., Rueschemeyer, S.-A., \& Bekkering, H. (2010). How specifically are action verbs represented in the neural motor system: an fMRI study. NeuroImage, 53(4), 1318-25. doi: 10.1016/j.neuroimage.2010.06.071

van Dam, W. O., Speed, L. J., Lai, V., Vigliocco, G. \& Desai, R. H. (in press). Effects of motion speed in action representations. Brain and Language.

Wang, J., Conder, J. A., Blitzer, D. N., \& Shinkareva, S. V. (2010). Neural representation of abstract and concrete concepts: A meta-analysis of neuroimaging studies. Human Brain Mapping, 31(10), 1459-1468. doi: 10.1002/hbm.20950

Weintraub, D., Moberg, P. J., Culbertson, W. C., Duda, J. E., Katz, I. R., \& Stern, M. B. (2005). Dimensions of Executive Function in Parkinson's Disease. Dementia and Geriatric Cognitive Disorders, 20(2-3), 140-144. doi: 10.1159/000087043

Xu, D., Cole, M. H., Mengersen, K., Silburn, P. A., Qiu, F., Graepel, C., \& Kerr, G. K. (2014). Executive Function and Postural Instability in People with Parkinson's Disease. Parkinson's Disease, 2014, 1-8. doi: 10.1155/2014/684758

York, C., Olm, C., Boller, A., McCluskey, L., Elman, L., Haley, J., Seltzer, E., Chahine, L., Woo, J., Rascovsky, K., McMillan, C., \& Grossman, M. (2014). Action verb comprehension in amyotrophic lateral sclerosis and Parkinson's disease. Journal of Neurology, 261(6), 1073-1079. doi: 10.1007/s00415-014-7314-y 
A

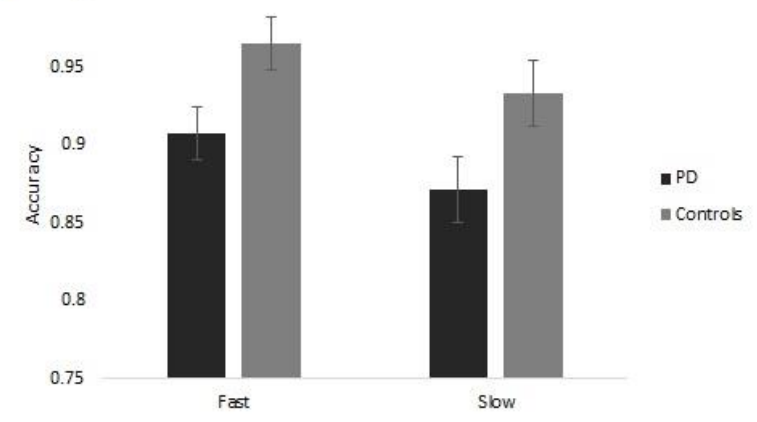

B

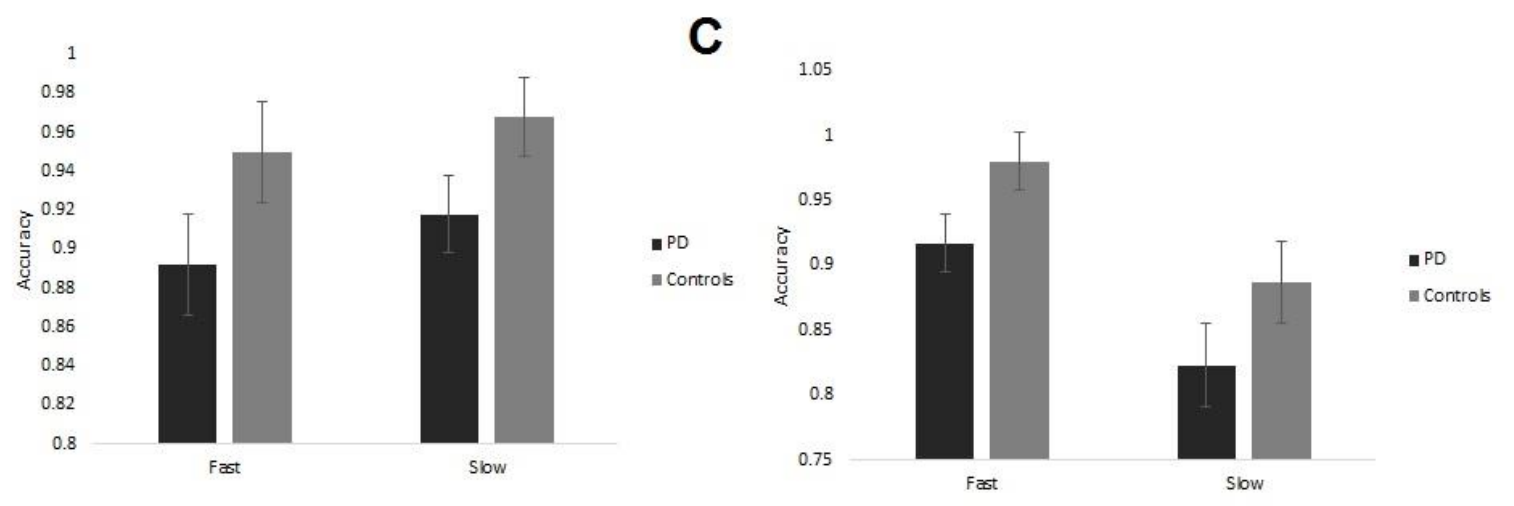


Figure 1.

A

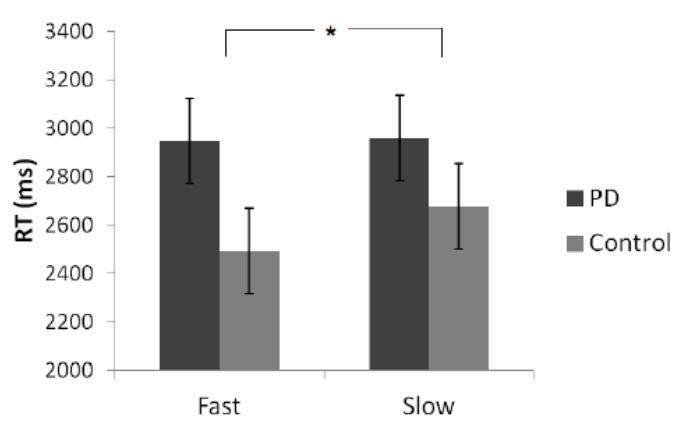

B

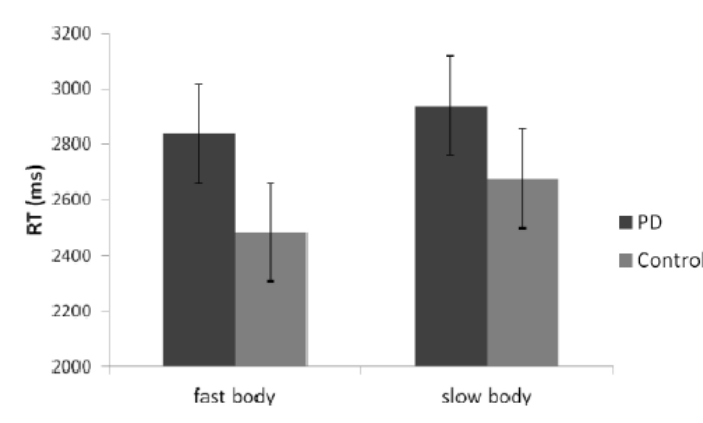

C

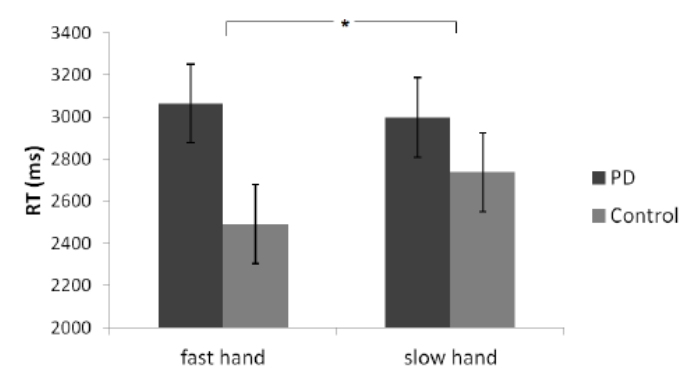

Figure 2. 


\begin{tabular}{|c|c|c|c|c|c|c|c|}
\hline & Gender & Age & MoCA & UPDRS & Yahr & since & medication \\
(mins)
\end{tabular}

\section{Table 1.}




\begin{tabular}{|l|c|c|c|}
\hline & Hand verbs & Full body verbs & Static verbs \\
\hline Arms* & $3.70(.56)$ & $2.39(.70)$ & $2.05(.46)$ \\
\hline Hands* & $3.91(.58)$ & $2.28(.68)$ & $2(.47)$ \\
\hline Legs* & $1.98(.38)$ & $3.53(.75)$ & $2.50(.74)$ \\
\hline Feet* & $1.99(.38)$ & $3.34(.68)$ & $2.27(.53)$ \\
\hline Torso & $2.50(.64)$ & $2.68(.51)$ & $2.47(.61)$ \\
\hline Effort & $2.89(.55)$ & $3.02(.75)$ & $2(.53)$ \\
\hline
\end{tabular}

\section{Table 2.}


Figure Legends

Figure 1. Average accuracy for semantic similarity judgments overall (A), for full-body verbs (B) and for hand verbs (C).

Figure 2. Average response time for semantic similarity judgments overall (A), for full-body verbs (B) and for hand verbs (C).

Table Legends

Table 1. Individual patient information for gender, age (years), Montreal Cognitive Assessment $(\max =30)$, Unified Parkinson's Disease Scale $(\max =32)$, Hoen-Yahr stage $(\max =4)$ and years since diagnosis.

Table 2. Mean body part ratings for hand and full body verbs $(1=$ low, $5=$ high $)$ * indicates significant difference between full body verb and hand verb. 


\section{Appendix A}

Verbs and mean speed ratings

\begin{tabular}{|l|l|}
\hline \multicolumn{1}{|c|}{ Verb } & Mean speed rating \\
\hline Fast-full body & 3.71 \\
to advance & 6 \\
to bound & 6.17 \\
to dash & 6.14 \\
to hurry & 6 \\
to race & 6.71 \\
to shoot & 6.14 \\
to sprint & 6.83 \\
Fast - hand & 6.1 \\
to grab & 6.67 \\
to shove & 6.9 \\
to slap & 6.9 \\
to smack & 6.9 \\
\hline
\end{tabular}




\begin{tabular}{|c|c|}
\hline $\begin{array}{l}\text { to whack } \\
\text { Slow-full body }\end{array}$ & 6.1 \\
\hline to crawl & 2 \\
\hline to ramble & 2.86 \\
\hline to roam & 2.86 \\
\hline to shuffle & 2.26 \\
\hline to sneak & 2.83 \\
\hline to step & 3.43 \\
\hline to trek & 3.43 \\
\hline to wander & 3 \\
\hline Slow - hand & \\
\hline to brush & 2.8 \\
\hline to caress & 1.8 \\
\hline to carry & 3 \\
\hline to feel & 2.3 \\
\hline to handle & 2.67 \\
\hline to hug & 2.4 \\
\hline to roll & 3.2 \\
\hline to stroke & 1.9 \\
\hline Static verbs & \\
\hline to cease & \\
\hline to delay & \\
\hline to desist & \\
\hline to finish & \\
\hline to freeze & \\
\hline
\end{tabular}




\begin{tabular}{|l|l|}
\hline to halt \\
to hesitate \\
to kneel \\
to lie \\
to lounge \\
to pause \\
to perch \\
to poise \\
to pose \\
to recline \\
to stap \\
to stand \\
to settle \\
to remain \\
to repose
\end{tabular}


to suspend

to wait 